\title{
Cellulose aerogels decorated with multi-walled carbon nanotubes: preparation, characterization, and application for electromagnetic interference shielding
}

\author{
Jian LI (ه), Caichao WAN \\ Material Science and Engineering College, Northeast Forestry University, Harbin 150040, China
}

\begin{abstract}
Electromagnetic wave pollution has attracted extensive attention because of its ability to affect the operation of electronic machinery and endanger human health. In this work, the environmentally-friendly hybrid aerogels consisting of cellulose and multi-walled carbon nanotubes (MWCNTs) were fabricated. The aerogels have a low bulk density of $58.17 \mathrm{mg} \cdot \mathrm{cm}^{-3}$. The incorporation of MWCNTs leads to an improvement in the thermal stability. In addition, the aerogels show a high electromagnetic interference (EMI) $S E_{\text {total }}$ value of $19.4 \mathrm{~dB}$. Meanwhile, the absorption-dominant shielding mechanism helps a lot to reduce secondary radiation, which is beneficial to develop novel eco-friendly EMI shielding materials.
\end{abstract}

Keywords cellulose aerogels, carbon nanotubes, electromagnetic interference shielding, composites

\section{Introduction}

Cellulose aerogels consist of micro/nano-scale threedimensional networks, and have a great variety of extraordinary features, such as ultra-low density, large specific surface, high porosity, and superb thermal, sound and electrical insulation characteristics ${ }^{[1,2]}$, which are currently considered one of the most promising biomaterials in the field of plant products. Also, it is believed that their intertangled fibril networks and ample surface hydroxyl groups contribute to tightly immobilizing nanoparticles and effectively reducing agglomeration. Therefore, cellulose aerogels are extensively regarded as

Received October 19, 2015; accepted November 16, 2015

Correspondence: nefulijian@163.com appropriate templates for the creation of composites with functional properties ${ }^{[3]}$. Our previous reports have justified the cellulose aerogels that could serve as suitable environmentally benign templates to support well-dispersed nanoscale $\gamma-\mathrm{Fe}_{2} \mathrm{O}_{3}{ }^{[4]}$, cobalt ferrite ${ }^{[5]}$, anatase titania ${ }^{[6]}$ and rod-like $\mathrm{ZnO}^{[7]}$.

Carbon nanotubes (CNTs) are an alluring class of materials from both theoretical and applied viewpoints, which have attracted tremendous attention since the discovery by Iijima in 1991, due to their outstanding electrical, mechanical, and physicochemical properties ${ }^{[8,9]}$. It has been reported that single-walled carbon nanotubes (SWCNTs) (a single graphite plane rolled up into a cylinder) have an ultra-low electrical resistivity of $10^{-6} \Omega \cdot \mathrm{cm}^{[10]}$. While for multi-walled carbon nanotubes (MWCNTs) composed of an array of concentric cylinders, the value is $3 \times 10^{-5} \Omega \cdot \mathrm{cm}$, which reveals that CNTs might be better conductors than metals such as copper at room temperature. In addition, CNTs are mechanically extremely strong, with Young's modulus and breaking strain larger than $1 \mathrm{TPa}$ and $5 \%{ }^{[11]}$, respectively. Also CNTs have low mass density, large aspect ratio ( $>100)$, high thermal conductivity, and chemical stability. Therefore, these properties make CNTs uniquely promising for serving as excellent filling agents in polymers. So far, a large number of investigations have been undertaken extensively for synthesizing miscellaneous composites consisting of CNTs combined with various matrix polymers such as thermosetting epoxy ${ }^{[12]}$, polyethylene $(\mathrm{PE})^{[13]}$, polyethyleneimine $(\mathrm{PEI})^{[14]}$, polyethyleneoxide $(\mathrm{PEO})^{[15]}$, poly(methyl methacrylate) (PMMA $)^{[16]}$, polypropylene (PP $)^{[17]}$, poly (vinyl alcohol) $(\mathrm{PVA})^{[18]}$. However, with the intensification of environmental pollutions and the increasingly depletion of petrochemical resources, sustainability, industrial ecology, eco-efficiency, and green chemistry are guiding the development of the next generation of materials, products, and processes. Therefore, it is 
interesting and significant to develop new eco-friendly CNT-based materials using environmentally friendly matrixes (e.g., cellulose aerogels) to replace the above petrochemical-based polymers, and extend their applications.

In the present work, the cellulose aerogels were fabricated via an eco-friendly inexpensive cellulose solvent (namely $\mathrm{NaOH} /$ polyethylene glycol), and the surface was decorated with MWCNTs. The hybrid MWCNT/cellulose aerogels (MWCNT/CA) were characterized by scanning electron microscope (SEM), X-ray diffraction (XRD), and thermogravimetry (TG) analysis. Moreover, as an example of the potential applications, the composite was used as an eco-friendly electromagnetic interference (EMI) shielding material to block undesirable electromagnetic irradiation.

\section{Materials and methods}

\subsection{Materials}

Filter paper served as the cellulosic source, and was cut into pieces and then dried at $60^{\circ} \mathrm{C}$ for $24 \mathrm{~h}$ to remove most absorbed water prior to use. MWCNTs are provided by Shanghai Aladdin Industrial Inc., China. The diameters of the MWCNTs varied from 20 to $40 \mathrm{~nm}$, and the lengths from 1 to $2 \mu \mathrm{m}$. Other chemical reagents were purchased from Tianjin Kermel Chemical Reagent Co., Ltd. (China) and used without further purification.

\subsection{Synthesis of MWCNT/CA}

MWCNTs $(0.10 \mathrm{~g})$ and cetyltrimethyl ammonium bromide (CTAB, $0.79 \mathrm{~g}$ ) were added to $100 \mathrm{~mL}$ absolute ethanol, and the mixture was magnetically stirred for $30 \mathrm{~min}$ and then subjected to an ultrasonic treatment by a sonifier (Scientz Technology, JY99-IID $900 \mathrm{~W} / 20 \mathrm{kHz}$ ) for $3 \mathrm{~h}$. For the preparation of the pure cellulose aerogels (PCA), the technical processes including dissolution, freezing-thawing, regeneration, and freeze drying were conducted in sequence. Full details are provided in earlier publications ${ }^{[19,20]}$. The resultant cylindrical PCA sample (about $0.30 \mathrm{~g})$ was immersed in the aforementioned alcohol dispersion of MWCNTs $(30 \mathrm{~mL})$ for $1 \mathrm{~h}$. Subsequently, the beaker containing the sample and the dispersion was placed into an oven at $50^{\circ} \mathrm{C}$ for about $12 \mathrm{~h}$ to form a black MWCNT coating on the surface of cellulose aerogels. Repeating the immersion and drying processes three times, the hybrid MWCNT/CA was successfully fabricated. Furthermore, the powder-like MWCNT/CA sample was prepared by mixing the alcohol dispersion of MWCNTs with the cellulose aerogels powder instead of the cylindrical cellulose aerogels, and used for the measurements of XRD, TG, and EMI shielding effectiveness (EMI $\mathrm{SE})$.

\subsection{Characterizations}

SEM observation was performed with a Hitachi S4800 SEM. The XRD patterns were measured with an XRD instrument (D/max 2200, Rigaku) using Ni filtered $\mathrm{Cu} \mathrm{Ka}$ radiation $(\lambda=1.5406 \AA)$ at $40 \mathrm{kV}$ and $30 \mathrm{~mA}$. Scattered radiation was detected ranging from $5^{\circ}$ to $40^{\circ}$ at a scan rate of $4^{\circ} \cdot \mathrm{min}^{-1}$. TG analysis experiments were performed by a synchronous thermal analyzer (SDT-Q600, USA) from room temperature to $700^{\circ} \mathrm{C}$ at a rate of $10^{\circ} \mathrm{C} \cdot \mathrm{min}^{-1}$ under a nitrogen atmosphere. EMI SE was measured using a PNA$\mathrm{X}$ network analyzer (N5244a) at the frequency range of 8$12 \mathrm{GHz}$ (X-band). The measured samples were prepared by uniformly mixing $40 \%$ of powder-like MWCNT/CA (or PCA) with $60 \%$ of paraffin wax (w/w). The mixture was then pressed into a toroidal shaped mold ( $\Phi_{\text {in }}: 3.0 \mathrm{~mm}$, $\Phi_{\text {out }}: 7.0 \mathrm{~mm}, H: 2.0 \mathrm{~mm}$ ).

\section{Results and discussion}

\subsection{Morphology and crystal structure of MWCNT/CA}

The morphology of MWCNT/CA was observed by SEM. It can be clearly seen in Fig. 1a that the fiber structures with
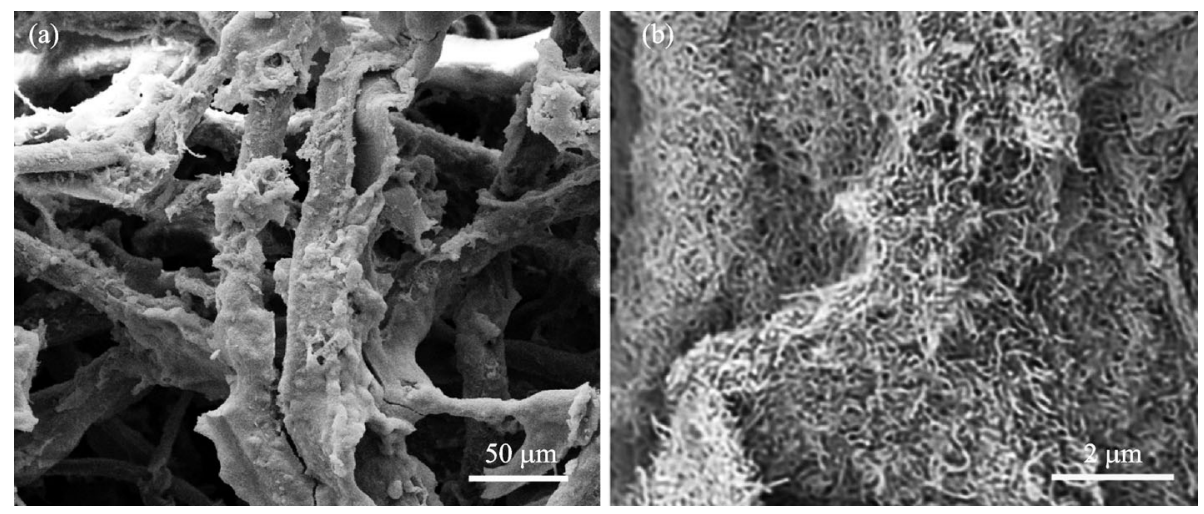

Fig. 1 Low-magnification (a) and high-magnification (b) SEM images of MWCNT/CA, respectively 
diameters of several microns are interlaced with each other. The porous network structure results in an extremely low bulk density of $58.17 \mathrm{mg} \cdot \mathrm{cm}^{-3}$, which was calculated by dividing the mass by the volume measured by a micrometer caliper. In addition, from the high-magnification SEM image of MWCNT/CA (Fig. 1b), it can be found that the surface of the fiber structures is coated with abundant dense MWCNTs, indicating that the drying process can effectively uniformly deposit the MWCNTs on the surface of the aerogels. This compact MWCNT coating may possibly contribute to reinforcing the thermal stability and EMI shielding ability of the composite.

The crystal structure of MWCNT/CA and PCA was investigated by XRD, and the resulting XRD patterns are presented in Fig. 2. For PCA, the diffraction peaks at around $12.4^{\circ}, 20.2^{\circ}$ and $21.9^{\circ}$ originate from the $(1 \overline{1} 0)$, (110), and (200) planes of cellulose II crystal structure ${ }^{[21]}$. For MWCNT/CA, the cellulose characteristic peaks of $(1 \overline{1} 0)$ and (110) planes at around $12.6^{\circ}$ and $20.5^{\circ}$ were still maintained, revealing that the immersion and drying processes did not seriously damage the cellulose crystal structure. In addition, the sharp XRD peak at $21.4^{\circ}$ is assigned to the (114) plane of $\mathrm{CTAB}^{[22]}$, which significantly fades the (200) peak of cellulose structure. Moreover, the peak at $24.5^{\circ}$ is possibly derived from the mixture

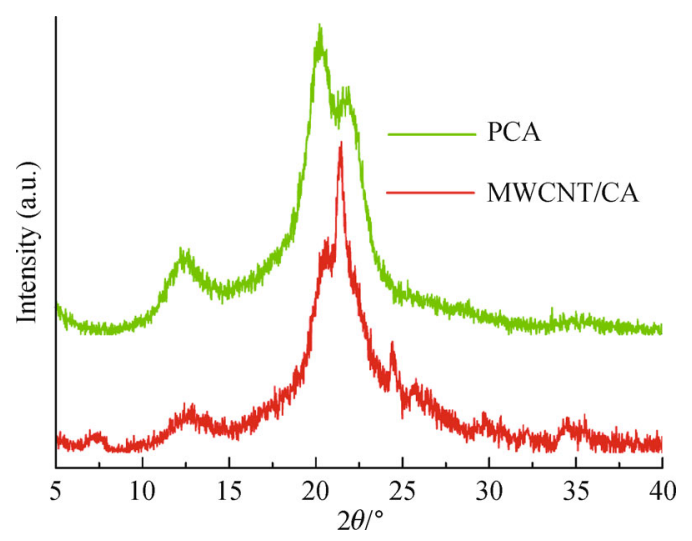

Fig. 2 XRD patterns of MWCNT/CA and PCA



of (002) peak of MWCNT and (020) peak of CTAB ${ }^{[23]}$. Apart from the surfactant (i.e., CTAB), the XRD pattern of the composite shows the presence of both components of MWCNT and cellulose, indicating a favorable combination.

\subsection{Thermal stability of MWCNT/CA}

For the investigation of the influence of the incorporation of MWCNT on the thermal stability of the composite, the thermal property of MWCNT/CA was measured by TG and derivative TG, and compared with that of PCA. As shown in Fig. 3a, apart from the small weight losses below $150^{\circ} \mathrm{C}$ due to the evaporation of adsorbed water, there was only a severe quality reduction stage for both samples occurring at around $270^{\circ} \mathrm{C}$ to $360^{\circ} \mathrm{C}$. The decomposition resulted from the depolymerization and decomposition of glucose units in cellulose ${ }^{[24]}$. The subsequent slow mass loses above $360^{\circ} \mathrm{C}$ are ascribed to the gradual oxidation and carbonization of cellulose. According to the TG plots, the weight loss during the whole pyrolysis process was around $81.8 \%$ and $98.9 \%$ for MWCNT/CA and PCA, respectively, which indicates that the proportion of MWCNT in MWCNT/CA can be roughly calculated as 17.1\%. In addition, as shown in Fig. $3 \mathrm{~b}$, the strong exothermic peak of MWCNT/CA centers at $345.6^{\circ} \mathrm{C}$, $8.0^{\circ} \mathrm{C}$ higher than that of PCA $\left(337.6^{\circ} \mathrm{C}\right)$. The increment reveals that the strong interaction between the cellulose and the MWCNTs makes contribution to the improvement in the thermal stability of the composite.

\subsection{EMI ability of MWCNT/CA}

In recent years, the pollution from EMI (especially in Xband) has attracted extensive attention because of its ability to affect the operation of electronic machines and endanger human health ${ }^{[25]}$. Therefore, the demand for eco-friendly, lightweight, and effective EMI shielding materials is extremely urgent. It is well-known that CNTs have huge potential for EMI shielding due to their excellent electrical properties, high aspect ratio, and high strength and

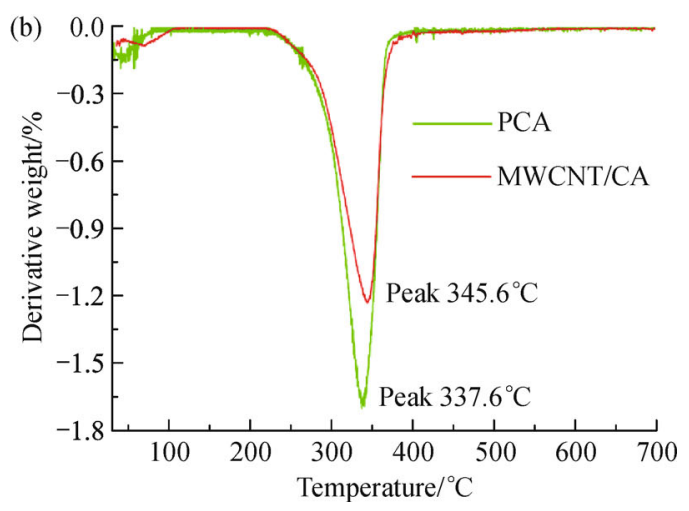

Fig. 3 TG (a) and derivative TG (b) curves of MWCNT/CA and PCA, respectively 
modulus ${ }^{[26]}$. Consequently, it is worthwhile to study the application potential of the CNT-based composites (i.e., MWCNT/CA) in EMI shielding.

As shown in Fig. 4, EMI SE includes the shielding effectiveness due to reflection $\left(S E_{\mathrm{R}}\right)$, shielding effectiveness due to absorption $\left(S E_{\mathrm{A}}\right)$, and shielding effectiveness due to multiple reflection $\left(S E_{\mathrm{M}}\right)$, which can be calculated through the following equations ${ }^{[27,28]}$ :

$$
\begin{gathered}
S E_{\text {total }}(\mathrm{dB})=10 \log \frac{P_{i}}{P_{t}}=S E_{\mathrm{A}}+S E_{\mathrm{R}}+S E_{\mathrm{M}} \\
S E_{\mathrm{R}}=-10 \log \left(1-\left|S_{11}\right|^{2}\right) \\
S E_{\mathrm{A}}=-10 \log \left[\left|S_{12}\right|^{2} /\left(1-\left|S_{11}\right|^{2}\right)\right]
\end{gathered}
$$

where $P_{\mathrm{i}}$ and $P_{\mathrm{t}}$ are the incident power and the transmitted power. When $S E_{\text {total }}>10 \mathrm{~dB}, S E_{\mathrm{M}}$ can be negligible. $\left|S_{12}\right|$ and $\left|S_{11}\right|$ are the scattering parameters ( $S$-parameters) of the two-port vector network analyzer system.

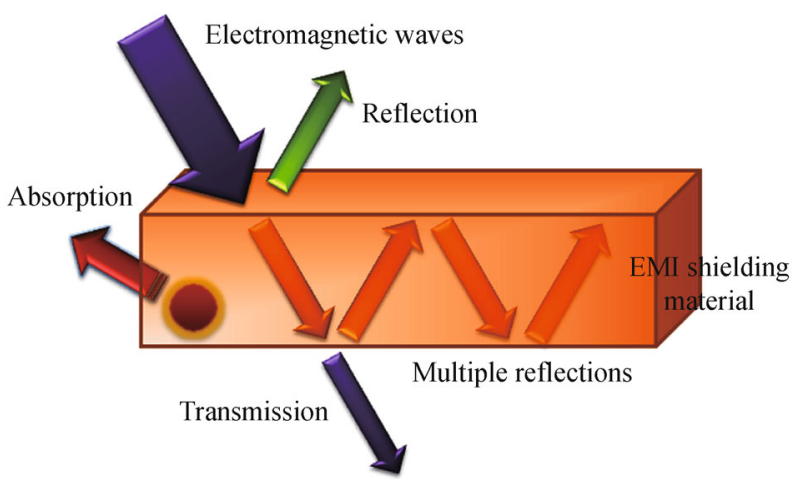

Fig. 4 Schematic diagram of major mechanisms for EMI shielding

In Fig. 5, PCA displays negligible fluctuation from 0 to $0.7 \mathrm{~dB}$ with the variation of frequency due to a shortage of effective groups or components that can interact with the

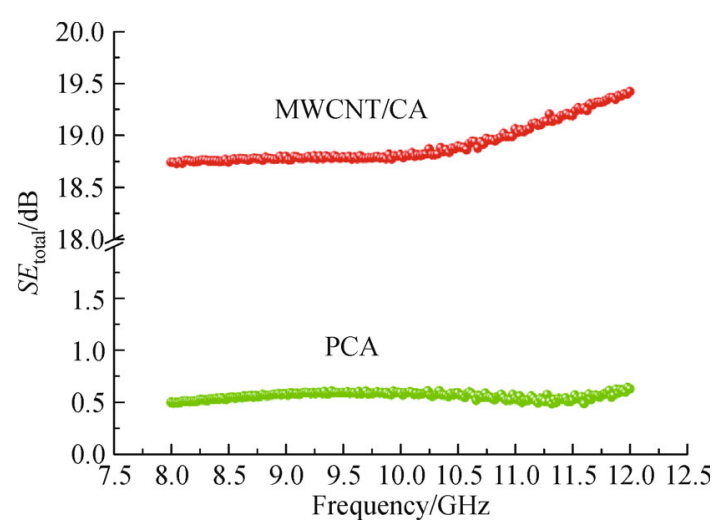

Fig. $5 S E_{\text {total }}$ of MWCNT/CA and PCA as a function of frequency

electromagnetic field ${ }^{[29]}$. For PCA coated with the MWCNTs (i.e., MWCNT/CA), a striking enhancement in $S E_{\text {total }}$ was found, which is ascribed to the extraordinary electromagnetic characteristics of CNTs. The $S E_{\text {total }}$ value reached as high as about $19.4 \mathrm{~dB}$, close to the commercially achievable level $(>20 \mathrm{~dB})^{[30]}$. Furthermore, additional approaches may possibly help to achieve further substantial improvements in the $S E_{\text {total }}$ of the composite, such as by purifying the MWCNTs for increasing electrical conductivity and inserting some conducting polymers (or other carbon-based materials) into the aerogels.

To clarify the EMI shielding mechanism of MWCNT/ CA, the EMI $S E_{\mathrm{R}}$ and $S E_{\mathrm{A}}$ of the composite in the 8 to 12 $\mathrm{GHz}$ range are present in Fig. 6. Comparison between the $S E_{\mathrm{R}}$ and $S E_{\mathrm{A}}$ suggests that $S E_{\mathrm{A}}$ is the dominant mechanism in EMI shielding. The $S E_{\mathrm{A}}$ value of MWCNT/CA approached 13.3 to $15.6 \mathrm{~dB}, 1.5$ to 3 times higher than that of $S E_{\mathrm{R}}(3.8$ to $5.4 \mathrm{~dB})$. Additionally, the absorptiondominant shielding mechanism is helpful in reducing secondary radiation, and is considered as one of the most critical factors for the exploitation of EMI shielding materials ${ }^{[31]}$. Also, the gradually upward trend of $S E_{\text {total }}$


Fig. $6 S E_{\mathrm{A}}(\mathrm{a})$ and $S E_{\mathrm{R}}(\mathrm{b})$ of MWCNT/CA and PCA as a function of frequency 
with increasing frequency (Fig. 5) is in agreement with the absorption-dominant shielding mechanism ${ }^{[32]}$.

\section{Conclusions}

A class of eco-friendly lightweight MWCNT/CA composite was prepared by repeatedly immersing the cellulose aerogels into the alcohol dispersion of MWCNTs and subsequent facile drying treatment. The SEM observations indicate that the MWCNTs were densely deposited on the surface of the aerogels, contributing to the improvement in the thermal stability of MWCNT/CA. In addition, the composite shows a high EMI $S E_{\text {total }}$ value of $19.4 \mathrm{~dB}$. The absorption-dominant shielding mechanism contributes to a reduction in secondary radiation, and is considered as one of the most critical factor for the exploitation of EMI shielding materials.

Acknowledgements This study was supported by the National Natural Science Foundation of China (31270590, 31470584).

Compliance with ethics guidelines Jian Li and Caichao Wan declare that they have no conflict of interest or financial conflicts to disclose.

This article does not contain any studies with human or animal subjects performed by any of the authors.

\section{References}

1. Liebner F, Potthast A, Rosenau T, Haimer E, Wendland M. Cellulose aerogels: highly porous, ultra-lightweight materials. Holzforschung, 2008, 62(2): 129-135

2. Sescousse R, Gavillon R, Budtova T. Aerocellulose from celluloseionic liquid solutions: preparation, properties and comparison with cellulose- $\mathrm{NaOH}$ and cellulose-NMMO routes. Carbohydrate Polymers, 2011, 83(4): 1766-1774

3. Pääkkö M, Vapaavuori J, Silvennoinen R, Kosonen H, Ankerfors M, Lindström T, Berglund L A, Ikkala O. Long and entangled native cellulose I nanofibers allow flexible aerogels and hierarchically porous templates for functionalities. Soft Matter, 2008, 4(12): 24922499

4. Wan $\mathrm{C}$, Li J. Facile synthesis of well-dispersed superparamagnetic $\gamma-\mathrm{Fe}_{2} \mathrm{O}_{3}$ nanoparticles encapsulated in three-dimensional architectures of cellulose aerogels and their applications for $\mathrm{Cr}$ (VI) removal from contaminated water. ACS Sustainable Chemistry \& Engineering, 2015, 3(9): 2142-2152

5. Wan C, Li J. Synthesis of well-dispersed magnetic $\mathrm{CoFe}_{2} \mathrm{O}_{4}$ nanoparticles in cellulose aerogels via a facile oxidative coprecipitation method. Carbohydrate Polymers, 2015, 134: 144-150

6. Wan C, Lu Y, Jin C, Sun Q, Li J. A facile low-temperature hydrothermal method to prepare anatase titania/cellulose aerogels with strong photocatalytic activities for rhodamine B and methyl orange degradations. Journal of Nanomaterials, 2015, 2015: 717016

7. Wan $\mathrm{C}$, Li J. Embedding $\mathrm{ZnO}$ nanorods into porous cellulose aerogels via a facile one-step low-temperature hydrothermal method. Materials \& Design, 2015, 83: 620-625

8. Baughman R H, Zakhidov A A, de Heer W A. Carbon nanotubesthe route toward applications. Science, 2002, 297(5582): 787-792

9. Berber S, Kwon Y K, Tománek D. Unusually high thermal conductivity of carbon nanotubes. Physical Review Letters, 2000, 84(20): 4613-4616

10. Li Q, Li Y, Zhang X, Chikkannanavar S B, Zhao Y, Dangelewicz A M, Zheng L, Doorn S K, Jia Q, Peterson D E, Arendt P N, Zhu Y. Structure-dependent electrical properties of carbon nanotube fibers. Advanced Materials, 2007, 19(20): 3358-3363

11. Zhang H, Wang Z, Zhang Z, Wu J, Zhang J, He J. Regeneratedcellulose/multiwalled-carbon nanotube composite fibers with enhanced mechanical properties prepared with the ionic liquid 1allyl-3-methylimidazolium chloride. Advanced Materials, 2007, 19 (5): 698-704

12. Hsieh T, Kinloch A, Taylor A, Kinloch I. The effect of carbon nanotubes on the fracture toughness and fatigue performance of a thermosetting epoxy polymer. Journal of Materials Science, 2011, 46(23): 7525-7535

13. Haggenmueller R, Fischer J E, Winey K I. Single wall carbon nanotube/polyethylene nanocomposites: nucleating and templating polyethylene crystallites. Macromolecules, 2006, 39(8): 2964-2971

14. Muñoz E, Suh D S, Collins S, Selvidge M, Dalton A B, Kim B G, Razal J M, Ussery G, Rinzler A G, Martínez M T, Baughman R H. Highly conducting carbon nanotube/polyethyleneimine composite fibers. Advanced Materials, 2005, 17(8): 1064-1067

15. Chatterjee T, Yurekli K, Hadjiev V G, Krishnamoorti R. Singlewalled carbon nanotube dispersions in poly(ethylene oxide). Advanced Functional Materials, 2005, 15(11): 1832-1838

16. Jin Z, Pramoda K, Xu G, Goh S H. Dynamic mechanical behavior of melt-processed multi-walled carbon nanotube/poly(methyl methacrylate) composites. Chemical Physics Letters, 2001, 337(1): 43-47

17. Chang T, Jensen L R, Kisliuk A, Pipes R, Pyrz R, Sokolov A. Microscopic mechanism of reinforcement in single-wall carbon nanotube/polypropylene nanocomposite. Polymer, 2005, 46(2): 439-444

18. Shaffer M S, Windle A H. Fabrication and characterization of carbon nanotube/poly(vinyl alcohol) composites. Advanced Materials, 1999, 11(11): 937-941

19. Li J, Wan C, Lu Y, Sun Q. Fabrication of cellulose aerogel from wheat straw with strong absorptive capacity. Frontiers of Agricultural Science and Engineering, 2014, 1(1): 46-52

20. Wan C, Lu Y, Jiao Y, Jin C, Sun Q, Li J. Fabrication of hydrophobic, electrically conductive and flame-resistant carbon aerogels by pyrolysis of regenerated cellulose aerogels. Carbohydrate Polymers, 2015, 118: 115-118

21. Nishiyama Y, Langan P, Chanzy H. Crystal structure and hydrogenbonding system in cellulose $\mathrm{I}_{\beta}$ from synchrotron X-ray and neutron fiber diffraction. Journal of the American Chemical Society, 2002, 124(31): 9074-9082

22. Bele M, Kodre A, Arčon I, Grdadolnik J, Pejovnik S, Besenhard J O. Adsorption of cetyltrimethylammonium bromide on carbon black from aqueous solution. Carbon, 1998, 36(7): 1207-1212

23. Chetty R, Kundu S, Xia W, Bron M, Schuhmann W, Chirila V, Brandld W, Reineckec T, Muhlera M. PtRu nanoparticles supported on nitrogen-doped multiwalled carbon nanotubes as catalyst for 
methanol electrooxidation. Electrochimica Acta, 2009, 54(17): 4208-4215

24. Wan J, Yan X, Ding J, Ren R. A simple method for preparing biocompatible composite of cellulose and carbon nanotubes for the cell sensor. Sensors and Actuators. B, Chemical, 2010, 146(1): 221225

25. Geetha S, Satheesh Kumar K, Rao C R, Vijayan M, Trivedi D. EMI shielding: Methods and materials-A review. Journal of Applied Polymer Science, 2009, 112(4): 2073-2086

26. Al-Saleh M H, Saadeh W H, Sundararaj U. EMI shielding effectiveness of carbon based nanostructured polymeric materials: a comparative study. Carbon, 2013, 60: 146-156

27. Liu X, Yin X, Kong L, Li Q, Liu Y, Duan W, Zhang L, Cheng L. Fabrication and electromagnetic interference shielding effectiveness of carbon nanotube reinforced carbon fiber/pyrolytic carbon composites. Carbon, 2014, 68: 501-510

28. Hao X, Yin X, Zhang L, Cheng L. Dielectric, electromagnetic interference shielding and absorption properties of $\mathrm{Si}_{3} \mathrm{~N}_{4}-\mathrm{PyC}$ composite ceramics. Journal of Materials Science and Technology, 2013, 29(3): 249-254

29. Alimohammadi F, Gashti M P, Shamei A. Functional cellulose fibers via polycarboxylic acid/carbon nanotube composite coating. Journal of Coatings Technology and Research, 2013, 10(1): 123132

30. Song W L, Fan L Z, Cao M S, Lu M M, Wang C Y, Wang J, Chen T T, Li Y, Hou Z L, Liu J, Sun Y P. Facile fabrication of ultrathin graphene papers for effective electromagnetic shielding. Journal of Materials Chemistry. C, Materials for Optical and Electronic Devices, 2014, 2(25): 5057-5064

31. Chung D. Materials for electromagnetic interference shielding. Journal of Materials Engineering and Performance, 2000, 9(3): 350-354

32. Zhang H B, Zheng W G, Yan Q, Jiang Z G, Yu Z Z. The effect of surface chemistry of graphene on rheological and electrical properties of polymethylmethacrylate composites. Carbon, 2012, 50(14): 5117-5125 